\title{
Targeting Plasmodium ligands on mosquito salivary glands and midgut with a phage display peptide library
}

\author{
Anil K. Ghosh, Paulo E. M. Ribolla*, and Marcelo Jacobs-Lorena ${ }^{\dagger}$ \\ Department of Genetics, Case Western Reserve University, 10900 Euclid Avenue, Cleveland, OH 44106-4955
}

Communicated by John H. Law, University of Arizona, Tucson, AZ, September 18, 2001 (received for review June 20, 2001)

Despite vast efforts and expenditures in the past few decades, malaria continues to kill millions of persons every year, and new approaches for disease control are urgently needed. To complete its life cycle in the mosquito, Plasmodium, the causative agent of malaria, has to traverse the epithelia of the midgut and salivary glands. Although strong circumstantial evidence indicates that parasite interactions with the two organs are specific, hardly any information is available about the interacting molecules. By use of a phage display library, we identified a 12-aa peptide-salivary gland and midgut peptide 1 (SM1) - that binds to the distal lobes of the salivary gland and to the luminal side of the midgut epithelium, but not to the midgut surface facing the hemolymph or to ovaries. The coincidence of the tissues with which parasites and the SM1 peptide interact suggested that the parasite and peptide recognize the same surface ligand. In support of this hypothesis, the SM1 peptide strongly inhibited Plasmodium invasion of salivary gland and midgut epithelia. These experiments suggest a new strategy for the genetic manipulation of mosquito vectorial capacity.

$\mathbf{T}$ he mosquito is an obligatory vector for transmission of malaria. Development of Plasmodium in the mosquito is complex and includes the crossing of two epithelia: midgut and salivary gland (1). Circumstantial evidence suggests that crossing of the epithelia requires specific interactions between Plasmodium and epithelial surface molecules $(2,3)$. However, little is known about the nature of these interactions. Soon after the mosquito ingests an infected blood meal, gametocytes transform into gametes that mate, giving rise first to zygotes and then to motile ookinetes. Before reaching the midgut epithelium, the ookinete must traverse the peritrophic matrix (PM), a thick chitinous sheath that completely surrounds the blood meal (4-6). To cross this barrier, the parasite secretes a chitinase (7). A mosquito gut-specific chitinase may also play a role in this process (8). To cross the midgut, ookinetes first adhere on the surface of the microvilli and then penetrate the epithelial cells $(3,9,10)$. Although the receptors and ligands required for the invasion process have not been identified, Abs against the midgut epithelial cells can partially block ookinete invasion (11). After emerging into the hemolymph side of the midgut, the ookinete differentiates into an oocyst that grows, and about 10-14 days later, releases mature sporozoites into the hemocoel. Only a fraction of the hemocoelic sporozoites ultimately invades the salivary glands, indicating that this process is inefficient. Compelling evidence suggests that the interaction between sporozoites and salivary glands is specific. First, sporozoites invade the distal but not the proximal salivary gland lobes (12). Second, Plasmodium knowlesi sporozoites can invade salivary glands from Anopheles dirus but not from Anopheles freeborni (2). Third, mAbs that recognize salivary gland epitopes partially block sporozoite invasion $(13,14)$. Thus, it is apparent that progression of Plasmodium development in the mosquito critically depends on recognition of mosquito midgut and salivary gland epithelia.

Here we report on the identification of a 12-aa peptide [salivary gland and midgut peptide 1 (SM1)] that binds specif- ically to the luminal side of the midgut epithelium and to the distal lobe of the salivary glands. Significantly, SM1 inhibits Plasmodium invasion of the two organs, suggesting that the peptide may be useful for the genetic modification of mosquito vectorial capacity.

\section{Methods}

Phage Selection. A library consisting of random dodecapeptides fused to the $\mathrm{N}$ terminus of the major filamentous $\mathrm{f} 88.4$ phage coat protein VIII was used (15). For selection of phages that bind to salivary glands, 10 adult Anopheles gambiae females were dissected $30 \mathrm{~min}$ after injecting about $10^{11}$ phages into their hemocoels (Fig. 1a). For selection of phages that bind to the midgut lumen, phages $\left(1 \times 10^{15} / \mathrm{ml}\right)$ suspended in $1 \mathrm{mg} / \mathrm{ml}$ of gamma globulin/ $120 \mathrm{mM} \mathrm{NaCl} / 20 \mathrm{mM} \mathrm{NaHCO} 3$ were fed to the mosquitoes, and midguts were dissected $30 \mathrm{~min}$ later. Sheets were prepared from the dissected midguts by making a longitudinal incision (Fig. 1b). Glands or midgut sheets were washed extensively in $1 \% \mathrm{BSA}$ in saline, and the bound phages were eluted with $50 \mathrm{mM}$ glycine buffer $(\mathrm{pH} 2)$. The recovered phages were amplified in Escherichia coli, and the selection was repeated 3 more times by using the same procedures, except that a 100 -fold excess of (tetracycline-sensitive) wild-type phages was mixed with the recovered phages before injection or feeding to the mosquitoes. After the fourth round of selection, individual randomly picked phages were sequenced to determine the predicted amino acid sequence of the corresponding peptide inserts.

Organ ELISA. Dissected salivary glands, midgut sheets, and ovaries from sugar-fed Anopheles stephensi females (4 organs per tube) were fixed in $4 \%$ paraformaldehyde for $1 \mathrm{~h}$ at $4^{\circ} \mathrm{C}$, washed 5 times with cold saline, and incubated for $2 \mathrm{~h}$ at $4^{\circ} \mathrm{C}$ in saline containing $4 \%$ BSA. Samples were incubated with $2 \times 10^{8}$ phages (either recombinant SM1 or wild-type control) for $2 \mathrm{~h}$ at $4^{\circ} \mathrm{C}$ in $200 \mu \mathrm{l}$ of saline containing $2 \% \mathrm{BSA}$, washed 5 times with saline at room temperature, blocked with BSA as before, and incubated with a peroxidase-tagged anti-M13 Ab (1:1000 dilution; Amersham Pharmacia Biotech, catalog no. 27-9402) for $2 \mathrm{~h}$ at $4^{\circ} \mathrm{C}$. The organs were washed and incubated with $3,3^{\prime}, 5,5^{\prime}-$ tetramethyl-benzidine (Sigma) for $1 \mathrm{~h}$ at room temperature in presence of $\mathrm{H}_{2} \mathrm{O}_{2}$. The reaction was stopped with $25 \mu \mathrm{l}$ of $0.2 \mathrm{M}$ $\mathrm{HCl}$, and the $A_{450}$ of the supernatant was measured.

Histochemical Staining of Salivary Glands and Midguts. Salivary glands from sugar-fed An. stephensi females were fixed overnight

\footnotetext{
Abbreviation: SM1, salivary gland and midgut peptide 1.

*Present address: Departamento de Parasitologia, Instituto de Biociências, Universidade Estadual Paulista, 18618-000, Botucatu, Brazil.

†To whom reprint requests should be addressed. E-mail: mxj3@po.cwru.edu.

The publication costs of this article were defrayed in part by page charge payment. This article must therefore be hereby marked "advertisement" in accordance with 18 U.S.C. $\S 1734$ solely to indicate this fact.
} 
a) Salivary Glands

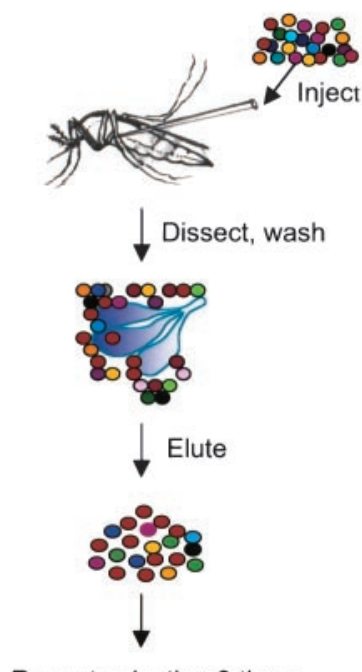

Repeat selection 3 times

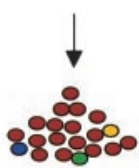

Enriched phage population b) Midguts

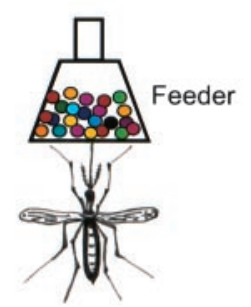

Dissect, wash

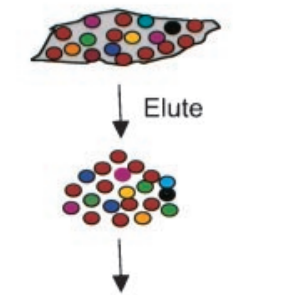

Repeat selection 3 times

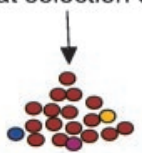

Enriched phage population

Fig. 1. Schematic illustration of the selection protocol for phages that bind either to salivary glands (a) or to the luminal side of the midgut epithelium (b).

at $4{ }^{\circ} \mathrm{C}$ in $4 \%$ paraformaldehyde, washed with saline, and incubated overnight at $4^{\circ} \mathrm{C}$ with blocking buffer (4\% BSA $/ 10 \%$ FBS). Glands were incubated for $2 \mathrm{~h}$ at $4^{\circ} \mathrm{C}$ with either the SM1 or a control peptide (100 $\mu \mathrm{g} / \mathrm{ml}$ of blocking buffer), both tagged at their $\mathrm{N}$ termini with a biotin residue. The control peptide had the ECVHLDDWVICT sequence. After washing in saline and incubating in blocking buffer for $2 \mathrm{~h}$, the bound peptides were detected by incubation for $1 \mathrm{~h}$ with an FITC-conjugated streptavidin solution in blocking buffer $(10 \mu \mathrm{g} / \mathrm{ml}$ final concentration; Molecular Probes, catalog no. S-869). Staining with 4',6diamidino-2-phenylindole (DAPI; $10 \mu \mathrm{g} / \mathrm{ml}$ final concentration; Molecular Probes, catalog no. DAPI-1306) was done following the manufacturer's instructions. For microscopic observation, glands were mounted in glycerol buffer plus Slowfade (Molecular Probes, catalog no. S-2828). Midgut sheets were prepared from An. stephensi females dissected $36 \mathrm{~h}$ after feeding on mouse blood. To remove background fluorescence, midgut sheets were treated with an ascending series of methanol (30,50, and 70\% for $5 \mathrm{~min}$ each, and $100 \%$ methanol for $15 \mathrm{~min}$ ) followed by a descending series and hydration with saline. Fixing and staining with FITC-streptavidin was as for salivary glands. The confocal image shown (Fig. 3i) is a composite of 18 optical sections.

Inhibition of Salivary Gland Invasion by Sporozoites. An. stephensi mosquitoes were fed on mice infected with Plasmodium berghei ANKA 2.34 strain and kept at $21^{\circ} \mathrm{C}$ for 24 days. Dissected midguts from infected mosquitoes were homogenized in M199 medium with a loose-fitting pestle, and the homogenate was centrifuged through glass wool to collect the released sporozoites. These were purified further by centrifugation at $5,000 \times g$ in a Ficoll (Amersham Pharmacia Biotech) density gradient (13). Sporozoites were mixed with phages or peptides to a final concentration of about 30,000 sporozoites and either $5 \times 10^{7}$ phages or $0.2 \mu \mathrm{g}$ of peptide per $0.5 \mu \mathrm{l}$ of M199 medium. This volume was injected into the hemolymph of each mosquito.

After $24 \mathrm{~h}$, salivary glands from the injected mosquitoes were dissected, homogenized in saline, and the number of sporozoites in each gland was determined by counting in a hemacytometer.

\section{Results and Discussion}

Phage Selection. A phage display library was used to search for ligands on the two mosquito epithelia-salivary gland and midgut - that were invaded by the malaria parasite. The library consists of 12-aa peptides $\left(\mathrm{XCX}_{8} \mathrm{CX}\right.$, where $\mathrm{X}$ is any amino acid and $\mathrm{C}$ is cysteine) fused to the $\mathrm{N}$ terminus of protein VIII, the major coat protein of the filamentous f88.4 $\mathrm{fd}$ bacteriophage. The library was produced from about $10^{9}$ different phages. To select phages displaying peptides with affinity to salivary glands, about $10^{11}$ phages were injected into the hemocoels of female mosquitoes (Fig. 1a). The injected phages were allowed to disperse for about $30 \mathrm{~min}$, after which the salivary glands were dissected and rinsed in saline to remove loosely bound phages. The attached phages were then eluted by incubation in low $\mathrm{pH}$ buffer. The recovered phages were amplified and used for further rounds of selection. A similar procedure was used to select phages that bind to the luminal side of the midgut epithelium, except that the phage library was fed to the mosquito (Fig. 1b). Surprisingly, phages displaying the same peptide, PCQRAIFQSICN, termed SM1, were recovered from the two selection procedures. After the fourth round of selection, over one-third of the phages recovered from salivary glands and almost half of the phages from midguts displayed SM1. Also, in an independent experiment where midguts were dissected at $24 \mathrm{~h}$ (instead of $30 \mathrm{~min}$ ) after mosquitoes fed on the library, 40/40 phages displayed the SM1 peptide (results not shown). As a control, about 40 random phages from the original library were sequenced. No two sequences were the same and none had the SM1 sequence, indicating that the library was not heavily biased toward any particular peptide sequence. SM1 has no significant homology to sequences in databases. In summary, three different in vivo selection experiments (one with salivary glands and two with midguts) led to the isolation of the same peptide, PC-

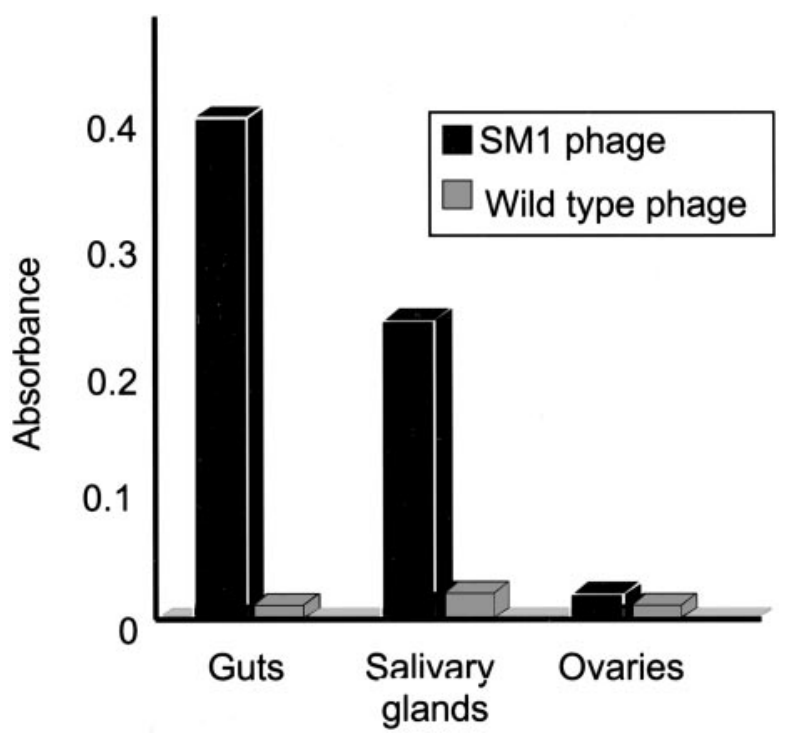

Fig. 2. Organ ELISA to determine affinity of the SM1 phage to salivary glands, midguts, and ovaries. Fixed organs were incubated with either SM1 or wild-type phages, and binding was detected with anti-phage Abs. The recombinant SM1 phage has high affinity to guts and salivary glands but not to ovaries. 

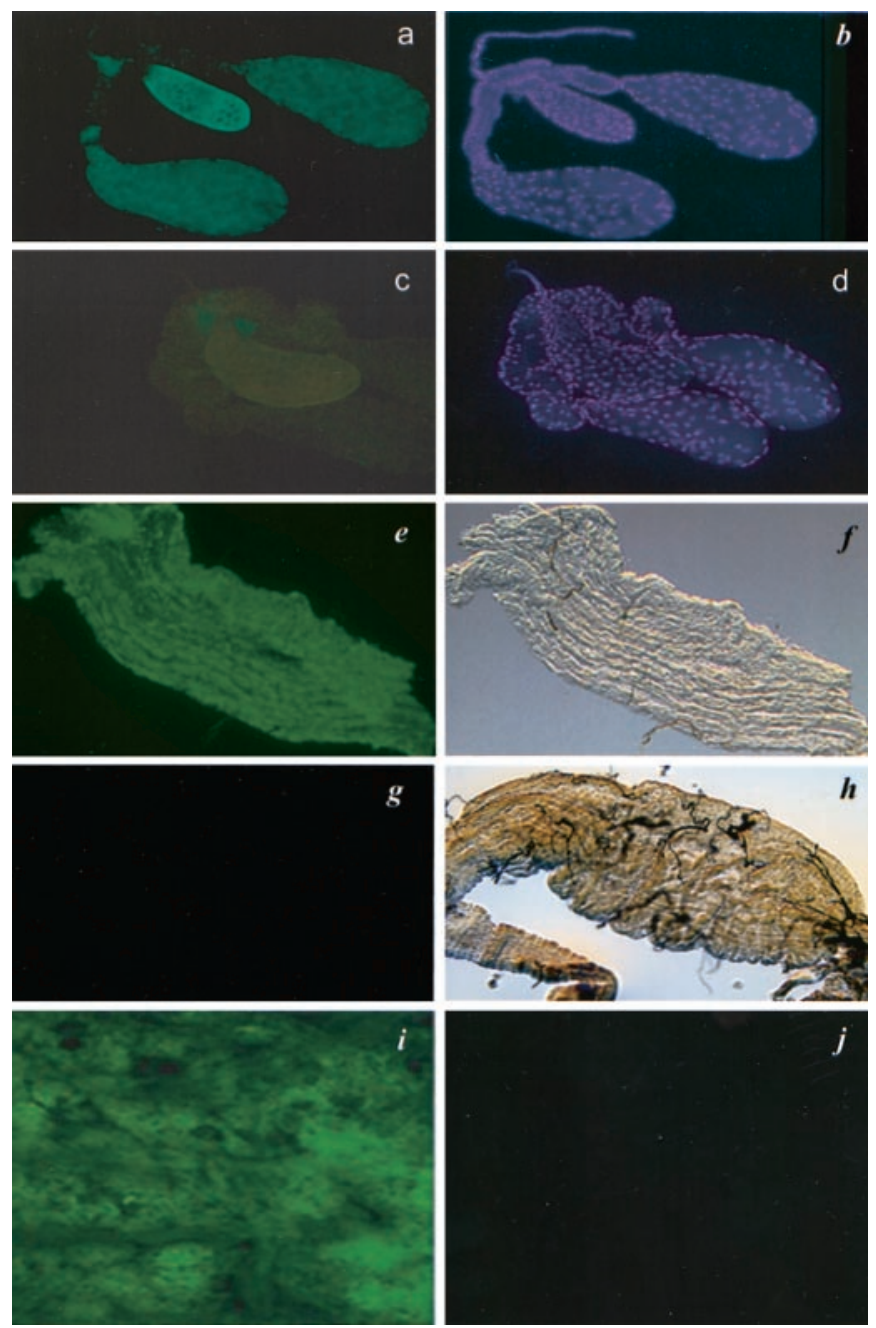

Fig. 3. Histochemical detection of SM1 binding to salivary glands and midguts. Fixed organs were incubated with a biotinylated peptide, washed, and binding was detected with fluorescently tagged avidin (a, c, e, $g$, and $i$ ). $b$ and $d$ show nuclear staining [4',6-diamidino-2-phenylindole (DAPI)], and panels $f$ and $h$ show light microscopic images of the same organs as those shown in the panels to their left. SM1 binds preferentially to salivary gland distal lobes (a), whereas the control peptide binds poorly to salivary glands (c). SM1 binds strongly to the luminal side of a gut sheet (e) but not to the outer (hemocoel) side of an intact midgut $(\mathrm{g})$. A more detailed image obtained with a confocal microscope reveals generalized binding of the peptide to all cells of a gut sheet $(i)$ but no detectable binding of the control peptide (j) to a similar gut sheet.

QRAIFQSICN, suggesting that salivary glands and midguts display common ligands.

Binding Specificity of the SM1 Phage. Specificity of SM1 binding was investigated by use of a modified ELISA. As shown in Fig. 2 , binding of the SM1 phage to midguts and salivary glands was significantly stronger than wild-type phage, whereas binding to ovaries was weak for either phage type. Because the surface area of each organ is different, only the ratio of SM1 to wild-type phage binding to a given organ can be compared. Similar specificity was observed when the binding of synthetic SM1 and control peptides (instead of phages) was compared (results not shown). Binding to salivary glands and midguts but not to ovaries indicates that peptide recognition is organ-specific. Although phage selection was done with An. gambiae mosquitoes, SM1 phage and peptide bound equally well to An. stephensi. This is the preferred mosquito for work with $P$. berghei (see below).
Table 1. Inhibition of sporozoite invasion by the SM1 phage or peptide

\begin{tabular}{llccc}
$\begin{array}{l}\text { Exp. } \\
\text { (phage or }\end{array}$ & $\begin{array}{l}\text { Phage or } \\
\text { peptide } \\
\text { peptide) }\end{array}$ & $\begin{array}{c}\text { Infected } \\
\text { salivary } \\
\text { injected }\end{array}$ & $\begin{array}{c}\text { Mean no. of } \\
\text { sporozoites per } \\
\text { salivary gland } \\
\text { pair (range) }\end{array}$ & $\begin{array}{c}\% \\
\text { Inhibition }\end{array}$ \\
\hline 1 (Phage) & Wild type & $6 / 7$ & $791(0-1100)$ & - \\
& SM1 & $4 / 8$ & $81(0-200)$ & 90 \\
2 (Peptide) & Control & $7 / 11$ & $531(0-1100)$ & - \\
& SM1 & $5 / 12$ & $59(0-150)$ & 89 \\
3 (Peptide) & Control & $3 / 4$ & $933(0-950)$ & - \\
& SM1 & $4 / 7$ & $64(0-200)$ & 93 \\
\hline
\end{tabular}

Purified sporozoites were mixed with phages or peptides and injected into the mosquito body cavity. At $24 \mathrm{~h}$ after injection, salivary glands were dissected, homogenized, and the no. of sporozoites that invaded each salivary gland pair was determined by counting in a hemacytometer. The sequence of the control peptide is given in Methods.

Binding Specificity of the SM1 Peptide. The spatial binding pattern of SM1 to salivary gland and midgut epithelia was investigated by using biotin-tagged synthetic peptides (Fig. 3). SM1 bound preferentially to the distal lobes of the salivary glands, whereas a control peptide that contained the two conserved cysteines but otherwise unrelated amino acids (see Methods) bound poorly (Fig. $3 a-d$ ). The SM1 peptide bound to the luminal but not to the outer surface of the midgut epithelium, further indicating specificity of binding (Fig. $3 e-h$ ). No selectivity of SM1 binding to any cell type of the midgut lumen was apparent (Fig. $3 i$ and $j$ ). Comparable results for salivary gland and midgut staining were obtained when phages (instead of peptides) were used.

SM1 Inhibits Sporozoite Invasion. Plasmodium sporozoites invade only the distal lobes of salivary glands (12). The coincidence between the patterns of SM1 binding and Plasmodium salivary gland invasion raised the possibility that the SM1 peptide and sporozoites recognize the same ligand(s). To test this hypothesis, $P$. berghei sporozoites were mixed with either SM1 or control peptide and injected into the hemocoels of An. stephensi females. $A n$. stephensi was used because efficiency of infection by $P$. berghei is much better than for An. gambiae. SM1, but not the control peptide, strongly inhibited salivary gland invasion (Table 1).

SM1 Inhibits Ookinete Invasion. Inhibition of midgut invasion by Plasmodium ookinetes was tested by feeding An. stephensi females either before (control) or after (experimental) injection of the SM1 peptide into the tail vein of an infected mouse. As shown in Table 2, SM1 (but not the control peptide) strongly inhibited oocyst formation, most likely by preventing ookinete invasion of the midgut. Importantly, mosquitoes that were fed on infected mice after peptide injection did not transmit the parasite when allowed to feed on naïve mice (Table 2). In control experiments, the SM1 peptide did not have any effect on ookinete differentiation in vitro. The number of ookinetes formed $\left(\approx 10^{5} / \mathrm{ml}\right)$ was unaffected by addition of SM1 or control peptide to either 100 or $200 \mu \mathrm{g} / \mathrm{ml}$ final concentration, indicating that this peptide is not toxic to the parasite. The present results suggest that the SM1 peptide inhibits parasite development in the mosquito by competing with Plasmodium for an essential salivary gland and midgut ligand(s). The small size of the peptide renders unlikely the possibility that inhibition is the result of steric hindrance after binding to an unrelated ligand(s).

In summary, we present evidence that the SM1 peptide severely hinders $P$. berghei development in An. stephensi, most likely by competing with a salivary gland and midgut ligand(s) required for Plasmodium invasion. It is likely that mosquito epithelial cell invasion is a multistep process, and that SM1 is 
Table 2. Inhibition of oocyst formation by the SM1 peptide Peptide Infected Mean oocysts/gut \%

\begin{tabular}{cccccc} 
Exp. & injected & guts/total & (range) & Inhibition & Transmission \\
\hline 1 & - & $5 / 5$ & $34(25-45)$ & - & ND \\
& SM1 & $0 / 5$ & 0 & 100 & ND \\
$1 a$ & - & $9 / 10$ & $9(0-90)$ & - & ND \\
& SM1 & $0 / 10$ & 0 & 100 & ND \\
2 & - & $23 / 25$ & $52(0-140)$ & - & YES \\
& SM1 & $6 / 25$ & $1(0-9)$ & 98 & NO \\
3 & - & $15 / 15$ & $77(5-301)$ & - & YES \\
& SM1 & $6 / 15$ & $2(2-23)$ & 96 & NO \\
4 & - & $9 / 10$ & $12(0-29)$ & - & ND \\
& SM1 & $1 / 10$ & $0(0-2)$ & 98 & ND \\
5 & - & $14 / 15$ & $21(0-44)$ & - & YES \\
& Control & $12 / 15$ & $21(0-130)$ & 3 & YES \\
\hline
\end{tabular}

For each experiment, An. stephensi were fed on an anesthetized $P$. bergheiinfected mouse followed by injection into its tail vein of $200 \mu \mathrm{g}$ of the SM 1 or control peptide dissolved in $200 \mu \mathrm{l}$ of saline. A second group of mosquitoes was fed on the mouse about 10 min after injection. Mosquitoes were kept at $21^{\circ} \mathrm{C}$, and the no. of oocysts per midgut was determined on days 7 (Exp. 1), 10 (Exp. 1a), or 15 (Exps. 2-5). To determine their ability to transmit the parasite, 12-15 mosquitoes from Exps. 2, 3, and 5 were allowed to feed on naive mice ( 2 mice per experiment) on day 25. The infection status of these mice was determined by microscopic observation of blood smears every other day for a period of up to 1 month. A "YES" in the last column denotes that both mice were infected by the mosquitoes, and a "NO" denotes that no infection was detected for at least 1 month after mosquito feeding. ND, not determined.

1. Ghosh, A., Edwards M. J. \& Jacobs-Lorena, M. (2000) Parasitol. Today 16, 196-201.

2. Rosenberg, R. (1985) Am. J. Trop. Med. Hyg. 34, 687-691.

3. Shahabuddin, M. \& Pimenta, P. F. (1998) Proc. Natl. Acad. Sci. USA 95, 3385-3389.

4. Jacobs-Lorena, M. \& Oo, M.-M. (1996) in Biology of Disease Vectors, eds Beaty, B. J. \& Marquardt, W. (Univ. Press of Colorado, Boulder), pp. 318-332.

5. Tellam, R. L. (1996) in Biology of the Insect Midgut, eds. Lehane, M. J. \& Billingsley, P. F. (Chapman \& Hall, London), pp. 86-114.

6. Shao, L., Devenport, M. \& Jacobs-Lorena, M. (2001) Arch. Insect. Biochem. Physiol. 47, 119-125.

7. Huber, M., Cabib, E. \& Miller, L. H. (1991) Proc. Natl. Acad. Sci. USA 88, $2807-2810$.

8. Shen, Z. \& Jacobs-Lorena, M. (1997) J. Biol. Chem. 272, 28895-28900. inhibiting only one essential step. The molecular identification of the ligand(s) and of the interacting Plasmodium protein(s) will provide clues about the mechanisms of cell invasion. Preliminary evidence suggests that the SM1 ligand(s) is not a carbohydrate. Importantly, our results predict that a mosquito that has been genetically modified to secrete this peptide into the hemocoel and/or into the midgut lumen will be refractory to malaria transmission. Finally, this report illustrates the power of phage display libraries as tools to investigate tissue surfaces in a whole-organism setting. This approach is generally applicable to a wide range of organisms and may provide effective means to examine research questions rendered inaccessible by conventional techniques.

We thank Drs. J. Scott and L. Bonnycastle (Simon Fraser University, Burnaby, Canada) for providing the phage display library. We also thank Drs. G. Dimopoulos and F. Kafatos (European Molecular Biology Laboratory, Heidelberg) and Drs. R. Sinden and G. Butcher (Imperial College, London) for providing $P$. berghei parasites. Expert technical assistance by J. Villalón and J. Snyder is gratefully acknowledged. This investigation received financial support from the United Nations Development Program/World Bank/World Health Organization Special Program for Research and Training in Tropical Diseases and from the National Institutes of Health. P.E.M.R. was supported by the Fundaçao de Amparo à Pesquisa do Estado de Sao Paulo (FAPESP)

9. Han, Y. S., Thompson, J., Kafatos, F. C. \& Barillas-Mury, C. (2000) EMBO J. 19, 6030-6040

10. Zieler, H. \& Dvorak, J. A. (2000) Proc. Natl. Acad. Sci. USA 97, 11516-11521.

11. Lal, A. A., Patterson, P. S., Sacci, J. B., Vaughan, J. A., Paul, C., Collins, W. S., Wirtz, R. A. \& Azad, A. F. (2001) Proc. Natl. Acad. Sci. USA 98, 5228-5233. (First Published April 17, 2001; 10.1073/pnas.10.1073/pnas.091447398)

12. Sterling, C. R., Aikawa, M. \& Vanderberg, J. P. (1973) J. Parasitol. 59, 593-605.

13. Barreau, C., Touray, M., Pimenta, P. F., Miller, L. H. \& Vernick, K. D. (1995) Exp. Parasitol. 81, 332-343.

14. Brennan, J. D., Kent, M., Dhar, R., Fujioka, H. \& Kumar, N. (2001) Proc. Natl. Acad. Sci. USA 97, 13859-13864. (First Published November 21, 2000; 10.1073/ pnas.10.1073/pnas.250472597)

15. Bonnycastle, L. L. C., Mehroke, J. S., Rashed, M., Gong, X. \& Scott, J. K. (1996) J. Mol. Biol. 258, 747-762. 